\title{
Irreducibility criteria for compositions and multiplicative convolutions of polynomials with integer coefficients
}

\author{
Anca Iuliana Bonciocat, Nicolae Ciprian Bonciocat and Mihai Cipu
}

Dedicated to Professor Mirela Ştefănescu

\begin{abstract}
We provide irreducibility criteria for multiplicative convolutions of polynomials with integer coefficients, that is, for polynomials of the form $h^{\operatorname{deg} f} \cdot f(g / h)$, where $f, g, h$ are polynomials with integer coefficients, and $g$ and $h$ are relatively prime. The irreducibility conditions are expressed in terms of the prime factorization of the leading coefficient of the polynomial $h^{\operatorname{deg} f} \cdot f(g / h)$, the degrees of $f, g, h$, and the absolute values of their coefficients. In particular, by letting $h=1$ we obtain irreducibility conditions for compositions of polynomials with integer coefficients.
\end{abstract}

\section{Introduction}

To decide whether the sum of two relatively prime polynomials is an irreducible polynomial, or in general, if a linear combination of two relatively prime polynomials is irreducible, is by no means an easy problem, and no general answer in this respect seems to be available. This problem may be raised for various classes of polynomials, like for instance polynomials with coefficients in an arbitrary unique factorization domain, or for multivariate

Key Words: multiplicative convolution of polynomials, resultants, irreducible polynomials.

2010 Mathematics Subject Classification: Primary 11R09; Secondary 11C08.

Received: 3 August, 2013.

Accepted: 28 August, 2013. 
polynomials over an arbitrary field. However, for some particular classes of such linear combinations of polynomials of the form $n_{1} f+n_{2} g$ some progress was recorded by using some information on the canonical decomposition of $n_{1}$ and $n_{2}$, or on the canonical decomposition of the coefficients of $f$ and $g$, or on their absolute values. Inspired by the work of Fried [8] and Langmann [9] in connection with Hilbert's irreducibility theorem, Cavachi [6] investigated the problem of the irreducibility of polynomials of the form $f(X)+p g(X)$, with $p$ prime, $f(X), g(X)$ relatively prime polynomials with rational coefficients, and $\operatorname{deg} f<\operatorname{deg} g$. Given two relatively prime polynomials $f(X), g(X) \in \mathbb{Q}[X]$ with $\operatorname{deg} f<\operatorname{deg} g$, an explicit bound $p_{0}$ was provided in [7] such that for all prime numbers $p>p_{0}$, the polynomial $f(X)+p g(X)$ is irreducible over $\mathbb{Q}$. In [4], explicit upper bounds have been derived for the number of factors over $\mathbb{Q}$ of a linear combination $n_{1} f(X)+n_{2} g(X)$, covering also the case $\operatorname{deg} f=$ $\operatorname{deg} g$. In [5] the same methods along with a Newton polygon argument have been used to find irreducibility conditions for linear combinations of the form $f(X)+p^{k} g(X)$. In [2] similar methods have been employed to study the irreducibility of some classes of compositions of polynomials, while in [3] the study focused on the irreducibility of some classes of multiplicative convolutions of polynomials, which offer considerably more flexibility to such irreducibility results, as they include linear combinations and compositions of polynomials as well.

Given two polynomials $g(X)=b_{0}+b_{1} X+\cdots+b_{n} X^{n}, h(X)=c_{0}+c_{1} X+$ $\cdots+c_{l} X^{l} \in \mathbb{Z}[X], b_{n} c_{l} \neq 0$, by a multiplicative convolution of $g$ and $h$ we understand any polynomial of the form

$$
\sum_{i=0}^{m} a_{i} g(X)^{i} h(X)^{m-i},
$$

with $a_{0}, a_{1}, \ldots, a_{m} \in \mathbb{Z}, m \geq 1, a_{0} a_{m} \neq 0$. If we associate to $a_{0}, a_{1}, \ldots, a_{m}$ the polynomial $f(X)=a_{0}+a_{1} X+\cdots+a_{m} X^{m}$, and assume that $h \neq 0$, then

$$
\sum_{i=0}^{m} a_{i} g(X)^{i} h(X)^{m-i}=h(X)^{m} f\left(\frac{g(X)}{h(X)}\right) .
$$

The aim of this paper is to complement some of the results in [3] and [2], by providing similar irreducibility conditions for the case when $a_{m}$ is divisible by a sufficiently large prime power. The irreducibility results that we will obtain for this kind of convolutions will be expressed in terms of the prime factorization of the leading coefficient of the polynomial $h^{\operatorname{deg} f} f(g / h)$, the degrees of $f, g$, $h$, and the absolute value of their coefficients. We use the same notation that was used in [3]. Given a polynomial $f(X)=a_{0}+\cdots+a_{m} X^{m} \in \mathbb{Z}[X]$ of degree $m \geq 0$, we let 


$$
H(f)=\max \left\{\left|a_{0}\right|, \ldots,\left|a_{m}\right|\right\} \quad \text { and } \quad L(f)=\sum_{i=0}^{m}\left|a_{i}\right|,
$$

and if $m>0$ we let

$$
H_{1}(f)=\max \left\{\left|a_{0}\right|, \ldots,\left|a_{m-1}\right|\right\} \quad \text { and } \quad L_{1}(f)=\sum_{i=0}^{m-1}\left|a_{i}\right| .
$$

Our main results refer to the case $\operatorname{deg} h<\operatorname{deg} g$ :

Theorem 1.1. Let $f(X)=a_{0}+a_{1} X+\cdots+a_{m} X^{m}, g(X)=b_{0}+b_{1} X+$ $\cdots+b_{n} X^{n}$ and $h(X)=c_{0}+c_{1} X+\cdots+c_{l} X^{l} \in \mathbb{Z}[X]$ be polynomials of degree $m, n$ and $l$ respectively, with $m \geq 1, n>l, a_{0} \neq 0$, and $g, h$ relatively prime. Put

$$
d=\max \left\{i: i<m \text { and } a_{i} \neq 0\right\} \text { and } \beta=1+\left[H_{1}(g)+H(h)\right] /\left|b_{n}\right| .
$$

Assume that $a_{m}=p^{k} q$ with $p$ a prime number, $q$ a non-zero integer, $p \nmid q a_{d} b_{n} c_{l}$ and $k$ is a positive integer prime to $(m-d)(n-l)$. If

$$
\left|a_{m}\right|>\sum_{i=0}^{d}\left|a_{i}\right| \cdot\left[|q|^{n}\left|b_{n}\right|^{m n} L(h(\beta X))\right]^{m-i}
$$

then the polynomial $h^{m} \cdot f(g / h)$ is irreducible over $\mathbb{Q}$. The same conclusion holds in the wider range

$$
\left|a_{m}\right|>\sum_{i=0}^{d}\left|a_{i}\right| \cdot\left[|q|^{\frac{n}{m}}\left|b_{n}\right|^{n} L(h(\beta X))\right]^{m-i},
$$

provided that $f$ is irreducible over $\mathbb{Q}$.

We also obtain the following irreducibility criterion, by replacing the hypothesis " $f$ irreducible" by a simple numerical condition.

Corollary 1.2. Let $f(X)=a_{0}+a_{1} X+\cdots+a_{m} X^{m}, g(X)=b_{0}+b_{1} X+$ $\cdots+b_{n} X^{n}$ and $h(X)=c_{0}+c_{1} X+\cdots+c_{l} X^{l} \in \mathbb{Z}[X]$ be polynomials of degree $m, n$ and $l$ respectively, with $m \geq 1, n>l, a_{0} \neq 0$, and $g, h$ relatively prime. Put

$$
d=\max \left\{i: i<m \text { and } a_{i} \neq 0\right\} \text { and } \beta=1+\left[H_{1}(g)+H(h)\right] /\left|b_{n}\right| .
$$


Assume that $a_{m}=p^{k} q$ with $p$ a prime number, $q$ a non-zero integer, $p \nmid q a_{d} b_{n} c_{l}$ and $k$ is a positive integer prime to $(m-d)(n-l)$. If

$$
\left|a_{m}\right|>\max \left\{\sum_{i=0}^{d}\left|a_{i}\right| \cdot|q|^{m-i}, \sum_{i=0}^{d}\left|a_{i}\right| \cdot\left[|q|^{\frac{n}{m}}\left|b_{n}\right|^{n} L(h(\beta X))\right]^{m-i}\right\},
$$

then the polynomial $h^{m} \cdot f(g / h)$ is irreducible over $\mathbb{Q}$.

One of the corollaries of the main result in [2] is the following irreducibility criterion for compositions of polynomials with integer coefficients.

Corollary 1.3. ([2, Corollary 4]) Let $f(X)=\sum_{i=0}^{m} a_{i} X^{i}$ and $g(X)=$ $\sum_{i=0}^{n} b_{i} X^{i}$ in $\mathbb{Z}[X]$ be non-constant polynomials of degree $m$ and $n$ respectively, with $a_{0} \neq 0$. If $a_{m}=p \cdot q$ with $p$ a prime satisfying

$$
p>\max \left\{|q|^{m-1} L_{1}\left(f\left(\frac{X}{|q|}\right)\right),|q|^{n-1}\left|b_{n}\right|^{m n} L_{1}\left(f\left(\frac{X}{|q|^{n / m}\left|b_{n}\right|^{n}}\right)\right)\right\},
$$

then the polynomial $f \circ g$ is irreducible over $\mathbb{Q}$.

By taking $h(X)=1$ in Corollary 1.2 we obtain the following irreducibility criterion for compositions of polynomials, that complements Corollary 4 in [2].

Corollary 1.4. Let $f(X)=a_{0}+a_{1} X+\cdots+a_{m} X^{m}$ and $g(X)=b_{0}+b_{1} X+$ $\cdots+b_{n} X^{n} \in \mathbb{Z}[X]$ be polynomials of degree $m \geq 1$ and $n \geq 1$ respectively, $a_{0} \neq 0$. Put

$$
d=\max \left\{i: i<m \text { and } a_{i} \neq 0\right\}
$$

and assume that $a_{m}=p^{k} q$ with $p$ a prime number, $q$ a non-zero integer, $p \nmid q a_{d} b_{n}$ and $k$ is a positive integer prime to $(m-d) n$. If

$$
\left|a_{m}\right|>\max \left\{\sum_{i=0}^{d}\left|a_{i}\right| \cdot|q|^{m-i}, \sum_{i=0}^{d}\left|a_{i}\right| \cdot\left[|q|^{\frac{n}{m}}\left|b_{n}\right|^{n}\right]^{m-i}\right\},
$$

then the polynomial $f \circ g$ is irreducible over $\mathbb{Q}$.

Perhaps a comparison with [3] is in place. For the sake of convenience, we quote a similar irreducibility criterion derived from the main result of [3].

Corollary 1.5. ([3, Corollary 1]) Let $f(X)=a_{0}+a_{1} X+\cdots+a_{m} X^{m}$, $g(X)=b_{0}+b_{1} X+\cdots+b_{n} X^{n}$ and $h(X)=c_{0}+c_{1} X+\cdots+c_{l} X^{l} \in \mathbb{Z}[X]$ be polynomials of degree $m, n$ and $l$ respectively, with $m \geq 1, n>l, a_{0} \neq 0, f$ 
irreducible over $\mathbb{Q}$, and $g, h$ relatively prime. Let $\beta=1+\left[H_{1}(g)+H(h)\right] /\left|b_{n}\right|$. If $a_{m}=p q$ with $p$ a prime satisfying

$$
\left|a_{m}\right|>\sum_{i=0}^{m-1}\left|a_{i}\right| \cdot\left[|q|^{n}\left|b_{n}\right|^{m n} L(h(\beta X))\right]^{m-i},
$$

then the polynomial $h^{m} \cdot f(g / h)$ is irreducible over $\mathbb{Q}$.

It is apparent that in Corollary 1.5 the hypothesis is less restrictive with regard to $p$, the requirement $p \nmid q a_{d} b_{n} c_{l}$ being added in Theorem 1.1. The condition that $k$ is coprime to $(m-d)(n-l)$ is automatically satisfied when $k=1$, which is the case dealt with in Corollary 1 from [3]. However, as the cofactor $q$ can be much bigger when applying Corollary 1.5 than when using Theorem 1.1, the latter result is applicable to polynomials $f$ having the leading coefficient $a_{m}$ somewhat smaller.

\section{Proof of the main results}

For the proof of Theorem 1.1 we need the following lemma from [1], which extends Capelli's Theorem to multiplicative convolutions of polynomials.

Lemma 2.1. Let $K$ be a field, $f, g, h \in K[X], f$ irreducible over $K, g$ and $h$ relatively prime, and $f(\alpha)=0$. If

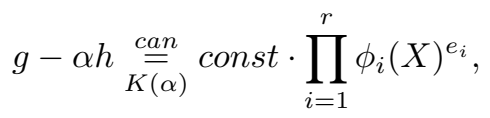

then

$$
h^{\operatorname{deg} f} \cdot f(g / h) \stackrel{\text { can }}{\bar{K}} \text { const } \cdot \prod_{i=1}^{r} N_{K(\alpha) / K} \phi_{i}(X)^{e_{i}} .
$$

In particular, the degree of every irreducible factor of $h^{\operatorname{deg} f} \cdot f(g / h)$ must be a multiple of $\operatorname{deg} f$.

Here $F \frac{\text { can }}{\bar{K}}$ const $\cdot \prod_{i=1}^{r} \phi_{i}(X)^{e_{i}}$ means that the $\phi_{i}$ 's are irreducible over $K$ and prime to each other. For a proof of this result in the case when $\operatorname{char}(K)=0$, which is relevant here, we refer the reader to [3].

Another result that will be needed in the proof of Theorem 1.1 is the following lemma in [5], whose proof relies on Newton's polygon method. 
Lemma 2.2. Let $u, v \in \mathbb{Z}[X]$ be two polynomials with $\operatorname{deg} v=n$ and $\operatorname{deg} u=n-d, d \geq 1$. Let also $p$ be a prime number that divides none of the leading coefficients of $u$ and $v$, and let $k$ be any positive integer prime to $d$. If $u(X)+p^{k} v(X)$ may be written as a product of two non-constant polynomials with integer coefficients, say $f_{1}$ and $f_{2}$, then one of the leading coefficients of $f_{1}$ and $f_{2}$ must be divisible by $p^{k}$.

We will also use the following basic lemma.

Lemma 2.3. Let $f(X)=a_{0}+a_{1} X+\cdots+a_{n} X^{n} \in \mathbb{C}[X]$ be a polynomial of degree $n$. If for a positive real $\delta$ we have

$$
\frac{\left|a_{n}\right|}{\delta^{n}}>L_{1}\left(f\left(\frac{X}{\delta}\right)\right)
$$

then all the roots of $f$ lie in the disk $|z|<\frac{1}{\delta}$.

Proof: Assume that $f$ has a root $\theta \in \mathbb{C}$ with $|\theta| \geq \frac{1}{\delta}$. Then we have

$$
0=\frac{|f(\theta)|}{\left|\theta^{n}\right|} \geq\left|a_{n}\right|-\frac{\left|a_{n-1}\right|}{|\theta|}-\cdots-\frac{\left|a_{0}\right|}{|\theta|^{n}} \geq\left|a_{n}\right|-\left|a_{n-1}\right| \delta-\cdots-\left|a_{0}\right| \delta^{n} .
$$

On the other hand, according to our hypothesis, we have $\frac{\left|a_{n}\right|}{\delta^{n}}>L_{1}\left(f\left(\frac{X}{\delta}\right)\right)$, so in fact we have $\left|a_{n}\right|>\left|a_{n-1}\right| \delta+\cdots+\left|a_{0}\right| \delta^{n}$, which contradicts (1).

We will now proceed with the proof of Theorem 1.1.

Proof of Theorem 1.1 Let $f(X), g(X)$ and $h(X)$ be as in the statement of the theorem. Let us assume to the contrary that $h^{m} \cdot f(g / h)$ factors as $h^{m} \cdot f(g / h)=F_{1} \cdot F_{2}$, with $F_{1}, F_{2} \in \mathbb{Z}[X]$, and $\operatorname{deg} F_{1} \geq 1, \operatorname{deg} F_{2} \geq 1$. Let $t_{1}, t_{2} \in \mathbb{Z}$ be the leading coefficients of $F_{1}$ and $F_{2}$, respectively. By comparing the leading coefficients in the equality

$$
h^{m} \cdot f(g / h)=a_{0} h^{m}+\cdots+a_{d} g^{d} h^{m-d}+a_{m} g^{m}=F_{1} \cdot F_{2}
$$

one finds that

$$
p^{k} q b_{n}^{m}=t_{1} \cdot t_{2} .
$$

By Lemma 2.2 with $a_{0} h(X)^{m}+\cdots+a_{d} g(X)^{d} h(X)^{m-d}$ instead of $u(X)$, and $q g(X)^{m}$ instead of $v(X)$, since $p \nmid q a_{d} b_{n} c_{l}$ and $k$ is prime to $(m-d)(n-l)$, we deduce that one of the leading coefficients of $F_{1}$ and $F_{2}$, say that of $F_{2}$, will be divisible by $p^{k}$. Then, in view of (2), it follows that $t_{1}$ will divide $q b_{n}^{m}$. In particular we have

$$
\left|t_{1}\right| \leq|q| \cdot\left|b_{n}\right|^{m}
$$


Let now $\bar{f}=h^{m} \cdot f(g / h)-a_{m} g^{m}$. Then $h^{m} \cdot f(g / h)=\bar{f}+a_{m} g^{m}$, and since $a_{0} \neq 0$, the polynomials $\bar{f}$ and $g^{m}$ are algebraically relatively prime.

Next, we will estimate the resultant $R\left(g^{m}, F_{1}\right)$. Since $g^{m}$ and $F_{1}$ are also algebraically relatively prime, $R\left(g^{m}, F_{1}\right)$ must be a non-zero integer number, so in particular we must have

$$
\left|R\left(g^{m}, F_{1}\right)\right| \geq 1 .
$$

Let $r=\operatorname{deg} F_{1} \geq 1$, and consider the decomposition of $F_{1}$, say

$$
F_{1}(X)=t_{1}\left(X-\theta_{1}\right) \cdots\left(X-\theta_{r}\right)
$$

with $\theta_{1}, \ldots, \theta_{r} \in \mathbb{C}$. Then

$$
\left|R\left(g^{m}, F_{1}\right)\right|=\left|t_{1}\right|^{m n} \prod_{1 \leq j \leq r}\left|g^{m}\left(\theta_{j}\right)\right| .
$$

Since each root $\theta_{j}$ of $F_{1}$ is also a root of $h^{m} \cdot f(g / h)$, we have

$$
g^{m}\left(\theta_{j}\right)=-\frac{\bar{f}\left(\theta_{j}\right)}{a_{m}}
$$

and moreover, since $\bar{f}$ and $g^{m}$ are relatively prime, $\bar{f}\left(\theta_{j}\right) \neq 0$ and $g^{m}\left(\theta_{j}\right) \neq 0$ for any $j \in\{1, \ldots, r\}$. Combining (5) and (6) we obtain

$$
\left|R\left(g^{m}, F_{1}\right)\right|=\frac{\left|t_{1}\right|^{m n}}{\left|a_{m}\right|^{r}} \cdot \prod_{1 \leq j \leq r}\left|\bar{f}\left(\theta_{j}\right)\right| .
$$

We now proceed to find an upper bound for $\left|\bar{f}\left(\theta_{j}\right)\right|$. In order to do this, we have to find an upper bound for the moduli of the roots of $f$. To this end, we first fix a positive real number $\delta$ such that $\left|a_{m}\right| / \delta^{m}>L_{1}(f(X / \delta))$. Later on we shall specify how to choose a convenient value of $\delta$. By Lemma 2.3 we see that all the roots $\lambda_{i}$ of $f$ will verify

$$
\left|\lambda_{i}\right|<\frac{1}{\delta}
$$

Let now $\theta_{1}, \ldots, \theta_{m n}$ be the roots of $h^{m} \cdot f(g / h)$. Since $g$ and $h$ are relatively prime, one has $h\left(\theta_{j}\right) \neq 0$ and $f\left(g\left(\theta_{j}\right) / h\left(\theta_{j}\right)\right)=0$ for $j=1, \ldots, m n$. Thus, for a given $\theta_{j}$, there exists $i_{j} \in\{1, \ldots, m\}$ such that $g\left(\theta_{j}\right) / h\left(\theta_{j}\right)=\lambda_{i_{j}}$, a root of $f$. By (8) we then have

$$
\left|\frac{g\left(\theta_{j}\right)}{h\left(\theta_{j}\right)}\right|<\frac{1}{\delta}
$$


Recall that $\bar{f}=a_{0} h^{m}+a_{1} g h^{m-1}+\cdots+a_{d} g^{d} h^{m-d}$. Using (9), we derive that

$$
\left|\bar{f}\left(\theta_{j}\right)\right|=\left|\sum_{i=0}^{d} a_{i} g\left(\theta_{j}\right)^{i} h\left(\theta_{j}\right)^{m-i}\right| \leq\left|h\left(\theta_{j}\right)\right|^{m} \cdot L_{1}(f(X / \delta)) .
$$

Combining now (7), (10) and (3) we deduce the following upper bound for $\left|R\left(g^{m}, F_{1}\right)\right|$ :

$$
\left|R\left(g^{m}, F_{1}\right)\right| \leq|q|^{m n}\left|b_{n}\right|^{m^{2} n} \cdot\left[\frac{\left|h\left(\theta_{j}\right)\right|^{m} L_{1}(f(X / \delta))}{\left|a_{m}\right|}\right]^{r} .
$$

The inequality (9) allows us to find also an upper bound for $\left|h\left(\theta_{j}\right)\right|$, as follows. We rewrite (9) as

$$
\delta\left|b_{0}+b_{1} \theta_{j}+\cdots+b_{n} \theta_{j}^{n}\right|<\left|c_{0}+c_{1} \theta_{j}+\cdots+c_{l} \theta_{j}^{l}\right|,
$$

which further gives

$$
\begin{aligned}
\delta\left|b_{n}\right| \cdot\left|\theta_{j}\right|^{n}< & \left(\left|c_{0}\right|+\delta\left|b_{0}\right|\right)+\left(\left|c_{1}\right|+\delta\left|b_{1}\right|\right)\left|\theta_{j}\right|+\cdots+\left(\left|c_{l}\right|+\delta\left|b_{l}\right|\right)\left|\theta_{j}\right|^{l} \\
& +\delta\left|b_{l+1}\right| \cdot\left|\theta_{j}\right|^{l+1}+\cdots+\delta\left|b_{n-1}\right| \cdot\left|\theta_{j}\right|^{n-1} \\
< & \left(H(h)+\delta H_{1}(g)\right) \cdot\left(1+\left|\theta_{j}\right|+\cdots+\left|\theta_{j}\right|^{n-1}\right) .
\end{aligned}
$$

Hence, for $\left|\theta_{j}\right|>1$ we obtain

$$
\delta\left|b_{n}\right| \cdot\left|\theta_{j}\right|^{n}<\left(H(h)+\delta H_{1}(g)\right) \cdot \frac{\left|\theta_{j}\right|^{n}-1}{\left|\theta_{j}\right|-1}<\left(H(h)+\delta H_{1}(g)\right) \cdot \frac{\left|\theta_{j}\right|^{n}}{\left|\theta_{j}\right|-1},
$$

that is

$$
\left|\theta_{j}\right|<1+\frac{H(h)+\delta H_{1}(g)}{\delta\left|b_{n}\right|} .
$$

Note that this inequality is trivially satisfied when $\left|\theta_{j}\right| \leq 1$. Denoting by $\gamma$ the right-hand side of (12), we find that for any root $\theta_{j}$ of $h^{m} \cdot f(g / h)$ it holds

$$
\left|h\left(\theta_{j}\right)\right|<L(h(\gamma X))
$$

which combined with (11) yields

$$
\left|R\left(g^{m}, F_{1}\right)\right| \leq|q|^{m n}\left|b_{n}\right|^{m^{2} n} \cdot\left[\frac{L(h(\gamma X))^{m} L_{1}(f(X / \delta))}{\left|a_{m}\right|}\right]^{r} .
$$

The next step is to choose $\delta$ so that inequalities (4) and (13) contradict each other. Since $\operatorname{deg} F_{1}=r \geq 1$, all it remains to prove is that our assumption 
on the size of $\left|a_{m}\right|$ will imply on the one hand $\left|a_{m}\right|>\delta^{m} L_{1}(f(X / \delta))$ for a suitable $\delta>0$, and on the other hand will force

$$
|q|^{m n}\left|b_{n}\right|^{m^{2} n} \cdot \frac{L(h(\gamma X))^{m} L_{1}(f(X / \delta))}{\left|a_{m}\right|}<1,
$$

or equivalently

$$
\left|a_{m}\right|>|q|^{m n}\left|b_{n}\right|^{m^{2} n} L(h(\gamma X))^{m} L_{1}(f(X / \delta)) .
$$

It will be therefore sufficient to have $\left|a_{m}\right|>\delta^{m} L_{1}(f(X / \delta))$ for a $\delta>0$ as small as possible satisfying $\delta^{m} \geq|q|^{m n}\left|b_{n}\right|^{m^{2} n} L(h(\gamma X))^{m}$, that is $\delta \geq$ $|q|^{n}\left|b_{n}\right|^{m n} L(h(\gamma X))$. Recalling the definition of $\gamma$, the last inequality reads

$$
\delta \geq|q|^{n}\left|b_{n}\right|^{m n} \sum_{i=0}^{l}\left|c_{i}\right|\left(1+\frac{H_{1}(g)}{\left|b_{n}\right|}+\frac{H(h)}{\delta\left|b_{n}\right|}\right)^{i} .
$$

A suitable candidate for $\delta$ is

$$
\delta_{0}=|q|^{n}\left|b_{n}\right|^{m n} \sum_{i=0}^{l}\left|c_{i}\right|\left(1+\frac{H_{1}(g)+H(h)}{\left|b_{n}\right|}\right)^{i} \geq 1,
$$

because

$$
1+\frac{H_{1}(g)+H(h)}{\left|b_{n}\right|} \geq 1+\frac{H_{1}(g)}{\left|b_{n}\right|}+\frac{H(h)}{\left|b_{n}\right| \delta_{0}} .
$$

By the definition of $\beta$, the positive real number $\delta_{0}$ just found coincides with $|q|^{n}\left|b_{n}\right|^{m n} L(h(\beta X))$, which proves that for

$$
\begin{aligned}
\left|a_{m}\right| & >\left[|q|^{n}\left|b_{n}\right|^{m n} L(h(\beta X))\right]^{m} \cdot L_{1}\left(f\left(\frac{X}{|q|^{n}\left|b_{n}\right|^{m n} L(h(\beta X))}\right)\right) \\
& =\sum_{i=0}^{d}\left|a_{i}\right| \cdot\left[|q|^{n}\left|b_{n}\right|^{m n} L(h(\beta X))\right]^{m-i}
\end{aligned}
$$

we actually have $\left|R\left(g^{m}, F_{1}\right)\right|<1$, a contradiction. Therefore $h^{m} \cdot f(g / h)$ must be irreducible over $\mathbb{Q}$, and this proves the first part of the theorem.

Assuming now that $f$ is irreducible over $\mathbb{Q}$, the proof goes as in the first part, except that according to Lemma 2.1, the degree of every irreducible factor of $h^{m} \cdot f(g / h)$ must be a multiple of $m$, so in particular we must have $\operatorname{deg}\left(F_{1}\right)=r \geq m$. In this case we need to prove that our assumption on the size of $p^{k}$ implies again $\left|a_{m}\right|>\delta^{m} L_{1}(f(X / \delta))$ for a suitable $\delta>0$, and at the same time it forces the inequality

$$
|q|^{n}\left|b_{n}\right|^{m n} \cdot \frac{L(h(\gamma X))^{m} L_{1}(f(X / \delta))}{\left|a_{m}\right|}<1,
$$


or equivalently

$$
\left|a_{m}\right|>|q|^{n}\left|b_{n}\right|^{m n} L(h(\gamma X))^{m} L_{1}(f(X / \delta)),
$$

which in view of (13) will contradict (4). It will be therefore sufficient to find a $\delta>0$ such that $\delta^{m} \geq|q|^{n}\left|b_{n}\right|^{m n} L(h(\gamma X))^{m}$, that is $\delta \geq|q|^{n / m}\left|b_{n}\right|^{n} L(h(\gamma X))$, which, recalling the definition of $\gamma$, reads

$$
\delta \geq|q|^{\frac{n}{m}}\left|b_{n}\right|^{n} \sum_{i=0}^{l}\left|c_{i}\right|\left(1+\frac{H_{1}(g)}{\left|b_{n}\right|}+\frac{H(h)}{\delta\left|b_{n}\right|}\right)^{i} .
$$

A suitable candidate for $\delta$ in this case is

$$
\delta_{1}=|q|^{\frac{n}{m}}\left|b_{n}\right|^{n} \sum_{i=0}^{l}\left|c_{i}\right|\left(1+\frac{H_{1}(g)+H(h)}{\left|b_{n}\right|}\right)^{i}=|q|^{\frac{n}{m}}\left|b_{n}\right|^{n} L(h(\beta X)) \geq 1,
$$

so the contradiction $\left|R\left(g^{m}, F_{1}\right)\right|<1$ follows now if

$$
\begin{aligned}
\left|a_{m}\right| & >\left[|q|^{\frac{n}{m}}\left|b_{n}\right|^{n} L(h(\beta X))\right]^{m} \cdot L_{1}\left(f\left(\frac{X}{|q|^{\frac{n}{m}}\left|b_{n}\right|^{n} L(h(\beta X))}\right)\right) \\
& =\sum_{i=0}^{d}\left|a_{i}\right| \cdot\left[|q|^{\frac{n}{m}}\left|b_{n}\right|^{n} L(h(\beta X))\right]^{m-i} .
\end{aligned}
$$

This completes the proof of the theorem.

Proof of Corollary 1.2 We apply Theorem 1.1 twice. On taking $g(X)=X$ and $h(X)=1$, we first note that if $f(X)=a_{0}+a_{1} X+\cdots+a_{m} X^{m} \in \mathbb{Z}[X]$ is a polynomial of degree $m \geq 1$, with $a_{0} \neq 0$, and $a_{m}=p^{k} q$ with $p$ a prime, $q$ a non-zero integer, $p \nmid q a_{d}$, and $k$ is a positive integer prime to $m-d$ and such that $p^{k}>|q|^{m-1} L_{1}(f(X /|q|))$, then $f$ is irreducible over $\mathbb{Q}$. Now by the second part of Theorem 1.1, the polynomial $h^{m} \cdot f(g / h)$ must be irreducible over $\mathbb{Q}$. So, if $p$ satisfies the hypothesis of Corollary 1.2, then both polynomials $f$ and $h^{m} \cdot f(g / h)$ will be irreducible over $\mathbb{Q}$.

Acknowledgements This research was partially supported by a grant of the Romanian Ministry of Education, CNCS-UEFISCDI, project PN-II-RUPD-2012-3-0656. The publication of this paper was supported by the grants of the Romanian Ministry of Education, CNCS-UEFISCDI, projects no. PNII-ID-WE- 2012-4-161 and PN-II-ID-WE-2012-4-169. 


\section{References}

[1] A.I. Bonciocat, N.C. Bonciocat, A Capelli type theorem for multiplicative convolutions of polynomials, Math. Nachr. 281 (2008), no. 9, 1240-1253.

[2] A.I. Bonciocat, A. Zaharescu, Irreducibility results for compositions of polynomials with integer coefficients, Monatsh Math. 149 (2006), no. 1, $31-41$.

[3] A.I. Bonciocat, N.C. Bonciocat, A. Zaharescu, On the number of factors of convolutions of polynomials with integer coefficients, Rocky Mountain J. Math. 38 (2008), no. 2, 417-431.

[4] N.C. Bonciocat, Upper bounds for the number of factors for a class of polynomials with rational coefficients, Acta Arith. 113. 2 (2004), 175-187.

[5] N.C. Bonciocat, Y. Bugeaud, M. Cipu, M. Mignotte, Irreducibility criteria for sums of two relatively prime polynomials, Int. J. Number Theory 9 (2013), no. 6, 1529-1539.

[6] M. Cavachi, On a special case of Hilbert's irreducibility theorem, J. Number Theory 82 (2000), no. 1, 96-99.

[7] M. Cavachi, M. Vâjâitu, A. Zaharescu, A class of irreducible polynomials, J. Ramanujan Math. Soc. 17 (2002), no. 3, 161-172.

[8] M. Fried, On Hilbert's irreducibility theorem, J. Number Theory 6 (1974), 211-231.

[9] K. Langmann, Der Hilbertsche Irreduzibilitätssatz und Primzahlfragen, J. Reine Angew. Math. 413 (1991), 213-219.

[10] A. Schinzel, Polynomials with Special Regard to Reducibility, in Encyclopedia of Mathematics and its Applications, Cambridge University Press (2000).

Anca Iuliana Bonciocat,

Simion Stoilow Institute of Mathematics of the

Romanian Academy, Research Unit 2,

P.O. Box 1-764, Bucharest 014700, Romania

Email: Anca.Bonciocat@imar.ro

Nicolae Ciprian Bonciocat,

Simion Stoilow Institute of Mathematics of the

Romanian Academy, Research Unit 5,

P.O. Box 1-764, Bucharest 014700, Romania

Email: Nicolae.Bonciocat@imar.ro 
Mihai Cipu,

Simion Stoilow Institute of Mathematics of the

Romanian Academy, Research Unit 5,

P.O. Box 1-764, Bucharest 014700, Romania

Email: Mihai.Cipu@imar.ro 